\title{
Predominance of PVL-negative community-associated methicillin-resistant Staphylococcus aureus sequence type 8 in newly diagnosed HIV-infected adults, Tanzania
}

\author{
Joel Manyahi ${ }^{1,2,3}$ (D) Sabrina J. Moyo ${ }^{1,3} \cdot$ Said Aboud $^{3} \cdot$ Nina Langeland ${ }^{1,2} \cdot$ Bjørn Blomberg $^{1,2}$
}

Received: 26 August 2020 / Accepted: 11 January 2021 / Published online: 14 February 2021

(C) The Author(s) 2021

\begin{abstract}
Difficult-to-treat infections caused by methicillin-resistant Staphylococcus aureus (MRSA) are of concern in people living with HIV infection as they are more vulnerable to infection. We aimed to identify molecular characteristics of MRSA colonizing newly diagnosed HIV-infected adults in Tanzania. Individuals newly diagnosed with HIV infection were recruited in Dar es Salaam, Tanzania, from April 2017 to May 2018, as part of the randomized clinical trial CoTrimResist (ClinicalTrials. gov identifier: NCT03087890). Nasal/nasopharyngeal isolates of Staphylococcus aureus were susceptibility tested by disk diffusion method, and cefoxitin-resistant isolates were characterized by short-reads whole genome sequencing. Four percent (22/537) of patients carried MRSA in the nose/nasopharynx. MRSA isolates were frequently resistant towards gentamicin (95\%), ciprofloxacin (91\%), and erythromycin (82\%) but less often towards trimethoprim-sulfamethoxazole (9\%). Seventy-three percent had inducible clindamycin resistance. Erythromycin-resistant isolates harbored ermC (15/18) and $L m r S(3 / 18)$ resistance genes. Ciprofloxacin resistance was mediated by mutations of the quinolone resistance-determining region (QRDR) sequence in the gyrA (S84L) and parC (S80Y) genes. All isolates belonged to the CC8 and ST8-SCCmecIV MRSA clone. Ninety-five percent of the MRSA isolates were spa-type t1476, and one exhibited spa-type t064. All isolates were negative for PantonValentine leucocidin (PVL) and arginine catabolic mobile element (ACME) type 1. All ST8-SCCmecIV-spa-t1476 MRSA clones from Tanzania were unrelated to the globally successful USA300 clone. Carriage of ST8 MRSA (non-USA300) was common among newly diagnosed HIV-infected adults in Tanzania. Frequent co-resistance to non-beta lactam antibiotics limits therapeutic options when infection occurs.
\end{abstract}

Joel Manyahi

manyahijoel@yahoo.com; joel.manyahi@uib.no

Sabrina J. Moyo

sabrina.moyo@uib.no

Said Aboud

aboudsaid@yahoo.com

Nina Langeland

nina.langeland@uib.no

Bjørn Blomberg

bjorn.blomberg@uib.no

1 Department of Clinical Science, University of Bergen, Bergen, Norway

2 National Advisory Unit for Tropical Infectious Diseases, Department of Medicine, Haukeland University Hospital, Bergen, Norway

3 Department of Microbiology and Immunology, Muhimbili University of Health and Allied Sciences, MUHAS, P.O. Box 65001, Dar es Salaam, Tanzania
Keywords Panton-Valentine leukocidin-negative $\cdot$ Sequence type 8 (ST8) - Methicillin-resistant Staphylococcus aureus (MRSA) · Human immunodficiency virus · Community · Tanzania

\section{Background}

Methicillin-resistant Staphylococcus aureus (MRSA) infections are difficult to treat and MRSA-bacteremia is associated with increased risk of fatal outcome [1]. Nasal colonization with MRSA is a risk factor for invasive disease [2] and a particular threat to HIV-infected individuals who are more susceptible to severe bacterial disease, including staphylococcal infections [3, 4]. Therefore, spread of community-associated methicillin-resistant Staphylococcus aureus (CA-MRSA) is of obvious concern [5] and particularly so in communities with large vulnerable population of HIV-infected people.

Understanding community transmission dynamics and spread of emerging CA-MRSA clonal populations is critical 
for the control and prevention of MRSA infection in HIV disease, and sequence typing can help shed light on the molecular epidemiology of circulating CA-MRSA population structures [6,7].

Despite the fact that nasal colonization with Staphylococcus aureus/MRSA is a risk factor for invasive infection in HIVinfected individuals, there are few countries in an African setting, Botswana, Ethiopia, Nigeria, and South Africa, which have reported carriage of MRSA among HIV-infected individuals [8-11]. These reports have focused on prevalence and risk factors of Staphylococcus aureus and MRSA using either conventional or PCR-based methods for detection and characterization of MRSA isolates. In Tanzania, no study has reported carriage of MRSA among HIV-infected individuals, and there is only one study from the Northern part of Tanzania which has described MRSA clone populations in clinical isolates, using whole genome sequencing technology [12]. Therefore, in Tanzania and many resource-limited countries in Africa, detailed information on the CA-MRSA population structure among HIV-infected individuals is scarce. As a result, measures for prevention and control of MRSA spread are insufficiently implemented in most health care facilities. This study used whole genome sequencing approach to provide data on nasal/ nasopharyngeal carriage of MRSA among newly diagnosed HIV-infected adults in a community setting in Tanzania. Additionally, we report sequence types, virulence genes, and phylogenetic analysis of the MRSA isolates.

\section{Methods and materials}

\section{Study participants}

A total of 537 individuals newly diagnosed with HIV infection were recruited at six sites: Amana, Mwananyamala, Temeke Regional Referral, PASADA, Mbagala, and Mnazi Mmoja hospitals in Dar es Salaam, from April 2017 to May 2018, as part of the randomized clinical trial CoTrimResist (ClinicalTrials.gov identifier: NCT03087890).

\section{Microbiological procedures}

\section{Specimen collection and bacterial culture}

Nasopharynx was swabbed first and, while retracting the swab anterior nares were swabbed. Nasopharyngeal/anterior nares swabs were collected by clinicians and transported to the microbiology laboratory (Muhimbili bacteriology research laboratory) immediately in a cool box at $4{ }^{\circ} \mathrm{C}$. Swabs were cultured on sheep blood agar for isolation of S. aureus. Isolates were identified by coagulase, mannitol fermentation and Staphaurex agglutination tests (Remel, Europe Ltd., Dartford, UK).
Antimicrobial susceptibility testing and screening for MRSA

Antimicrobial susceptibility testing was performed on Muller Hinton agar, and plates were incubated at $35^{\circ} \mathrm{C}$ for $16-18 \mathrm{~h}$. Kirby Bauer disk diffusion method was used for susceptibility testing for penicillin, gentamicin, erythromycin, trimethoprim-sulfamethoxazole, ciprofloxacin, and clindamycin; cefoxitin was also included for initial screening of MRSA (Oxoid, UK). Antimicrobial susceptibility testing was interpreted using Clinical and Laboratory Standards Institute (CLSI) guidelines [13]. The minimum inhibitory concentrations (MICs) for vancomycin and linezolid were determined by E-test (BioMérieux, Marcy-I’Etoile, France).

\section{Polymerase chain reaction (PCR) testing}

For all cefoxitin-resistant $S$. aureus isolates, we did real-time multiplex PCR targeting the $n u c$ and mecA genes to confirm the bacterial identity and presence of methicillin resistance, respectively. DNA was extracted by a rapid boiling procedure. PCR was performed using 2× QuantiTect Multiplex PCR NoROX Master Mix (Qiagen), and amplification was carried out on a Light Cycler 480 Instrument II (Roche Diagnostics, Mannheim, Germany). Primers used and PCR conditions have been described previously [14].

\section{Whole genome sequencing for MRSA}

All 22 MRSA isolates underwent whole genome sequencing (WGS). DNA extraction and whole genome sequencing was performed by MicrobesNG (MicrobesNG, Birmingham, UK). For WGS, Illumina HiSeq technology approach was used $(2 \times 250$ bp paired-end reads protocol) (Illumina, San Diego, CA, USA). Trimming and quality filtering of the sequencing reads were assembled using SPAdes and annotated in GenBank.

Assignment of multilocus sequence typing (MLST) was based on sequencing seven housekeeping genes (pta, $\operatorname{arcC}$, tpi, aroE, gmk, yqiL, and $g l p F$ ). Sequence type (ST) and clonal complex were determined by submission of sequence files to an online MLST database website (https://pubmlst.org/).

For identification of acquired antimicrobial resistance genes, virulence gene, SCCmec and spa-type, we used ResFinder v3.2, virulenceFinder 2.0, SCCmecFinder 1.2 and spaTyper 1.0 of the Center for Genomic Epidemiology GEE server (http://www.genomicepidemiology.org/). For analysis of interstrain whole genome single nucleotide polymorphism (SNPs), we used CSI phylogeny 1.4 (https://cge.cbs.dtu.dk/services/ CSIPhylogeny/).

We used Figtree (https://github.com/rambaut/figtree/ releases) to construct the phylogenetic tree. The whole genome SNPs tree included 22 ST8 from the present study, S. aureus USA300_FPR3757 (accession number CP000255), 
and 10 already well-characterized ST8 MRSA $[15,16]$. This Whole Genome Shotgun project for the present study has been deposited at DDBJ/ENA/GenBank under BioProject number PRJNA649684.

\section{Results}

\section{Bacterial isolates}

In this study, overall nasal/nasopharyngeal carriage rate of S. aureus was $14 \%$ (77/537), and $29 \%$ of the $S$. aureus isolates $(n=22)$ were resistant to cefoxitin, corresponding to a four percent MRSA carrier rate among all participants. All 22 isolates carried the mecA and nuc gene, confirming methicillinresistant $S$. aureus. Of 22 isolates, five were isolated from participants who reported having visited an outpatient clinic during the 1 month prior to specimen collection, and one had history of hospitalization during the month prior to specimen collection. Sixteen participants had not visited any outpatient clinics nor been hospitalized recently.

\section{Antimicrobial susceptibility patterns and genomic resistance traits}

All MRSA isolates were susceptible to vancomycin, linezolid, and clindamycin. The overall MRSA antimicrobial susceptibility profiles to other antibiotics are shown in Table 1 . Resistance to gentamicin, erythromycin, ciprofloxacin and trimethoprimsulfamethoxazole and were 95\% (21/22), 82\% (18/22), 91\% $(20 / 22)$ and $9 \%(2 / 22)$, respectively. Inducible clindamycin resistance was observed in $73 \%(16 / 22)$ of MRSA isolates. Phenotypic susceptibility patterns of all MRSA were highly concordant with genotypic findings (resistome and mutations in genome) (Table 1). All isolates carried blaZ gene mediating resistance to penicillin resistance. Resistance to aminoglycosides was mediated by the aac6'-aph2" gene. MRSA isolates resistant to erythromycin harbored ermC (15/18) and $L m r S$ (3/18) resistance genes (Table 1). MRSA resistance to ciprofloxacin was mediated by mutation of the quinolone resistancedetermining region (QRDR) sequence in the gyrA (S84L) and $\operatorname{parC}(\mathrm{S} 80 \mathrm{Y})$ genes. All MRSA isolates carried trimethoprim resistance gene $d f r G$, but resistance was not expressed phenotypically $(91 \%, 20 / 22$ susceptible to TMP-SMX). Fosfomycin resistance gene (FosB) and tetracycline resistance gene (tetK) were detected in $100 \%(22 / 22)$ and 55\% (12/22) of MRSA isolates, respectively.

\section{Virulence factors and immune evasion cluster genes}

Staphylococcal complement inhibitor (scn) and Staphylokinase ( $\mathrm{sak}$ ), which are among immune evasion cluster (IEC) genes, were detected in all MRSA isolates (Table 2).
None of the isolates carried other IEC genes, but one carried staphylococcal enterotoxin A.

Gamma-hemolysins ( $h \lg A, h \lg B$, and $h \lg C$ ) and leucocidin ED genes were detected in all MRSA isolates. Almost all isolates carried staphylococcal enterotoxin genes, with sej and ser genes being the most predominant. None of the MRSA harbored exfoliative toxin A/B or leucocidin S/F-PV genes. Only one isolates carried toxic shock syndrome toxin 1(tst $)$ gene.

\section{Population genetic structure of MRSA strains}

All MRSA isolates were ST8 and belonged to the CC 8 lineage (Table 1). All MRSA isolates were genotyped by spa type, the most predominant $(21 / 22,95 \%)$ was spa-type t1476, and one isolate was spa-type t064. On the other hand, genotype ST8SCCmecIV, which is a major CA-MRSA worldwide, were detected in MRSA isolates. All but one of the MRSA isolates belonged to SCCmecIV (2B\&5) subtype, and the remaining isolate had SCCmecIVa subtype.

Overall, genotype ST8-SCCmecIV-spa-t1476 was the predominant, and all isolates were negative for arginine catabolic mobile element (ACME) type 1 and Panton-Valentine leucocidin (PVL).

\section{Genetic relatedness among CC8/ST8 MRSA isolates}

We compared all $22 \mathrm{CC} 8 / \mathrm{ST} 8 \mathrm{MRSA}$ isolates from this study with already known ST8 MRSA (USA300) and one MRSA isolate from Tanzania (NZ_FMMT01000000), one from Gabon [16], and eight from the USA [15]. The percentage of reference genome (USA300_FPR3757 (accession number CP000255) covered by all isolates was $91.1 \%$, the size of the reference genome was 2,872,769, and 2,652,029 positions were found in all analyzed genomes. SNP analysis of 22 ST8 MRSA from the present study revealed SNPs differences from 0 to 988, showing high genetic diversity.

Phylogenetic analysis showed the presence of two distinct clades (Fig. 1). All MRSA isolates with ST8-SCCmecIV-spat1476 from Tanzania formed one clade, had SNPs difference between 1 and 199, and were unrelated to known ST8 clones. Three MRSA isolates had 2 SNPs differences (TZ6, TZ 380, and T207); these were from patients enrolled at different study sites. In one instance, 2 MRSA isolates had 3 SNPs differences (TZ 4 and TZ127). These two instances indicated recent spread of this isolates from a common source.

Two MRSA isolates with pairwise SNP difference of 102 SNPs, both ST8-spa-t064 from Tanzania (1 from present study and 1 from previous study), were clustered in another clade with USA300 isolates from the USA and Gabon, Africa. While these two ST8 MRSA isolates from Tanzania possessed SCCmecIVa and grouped with ST8 (USA300) from the USA, they were not closely related and had SNPs distances of more than 600 SNPs. 


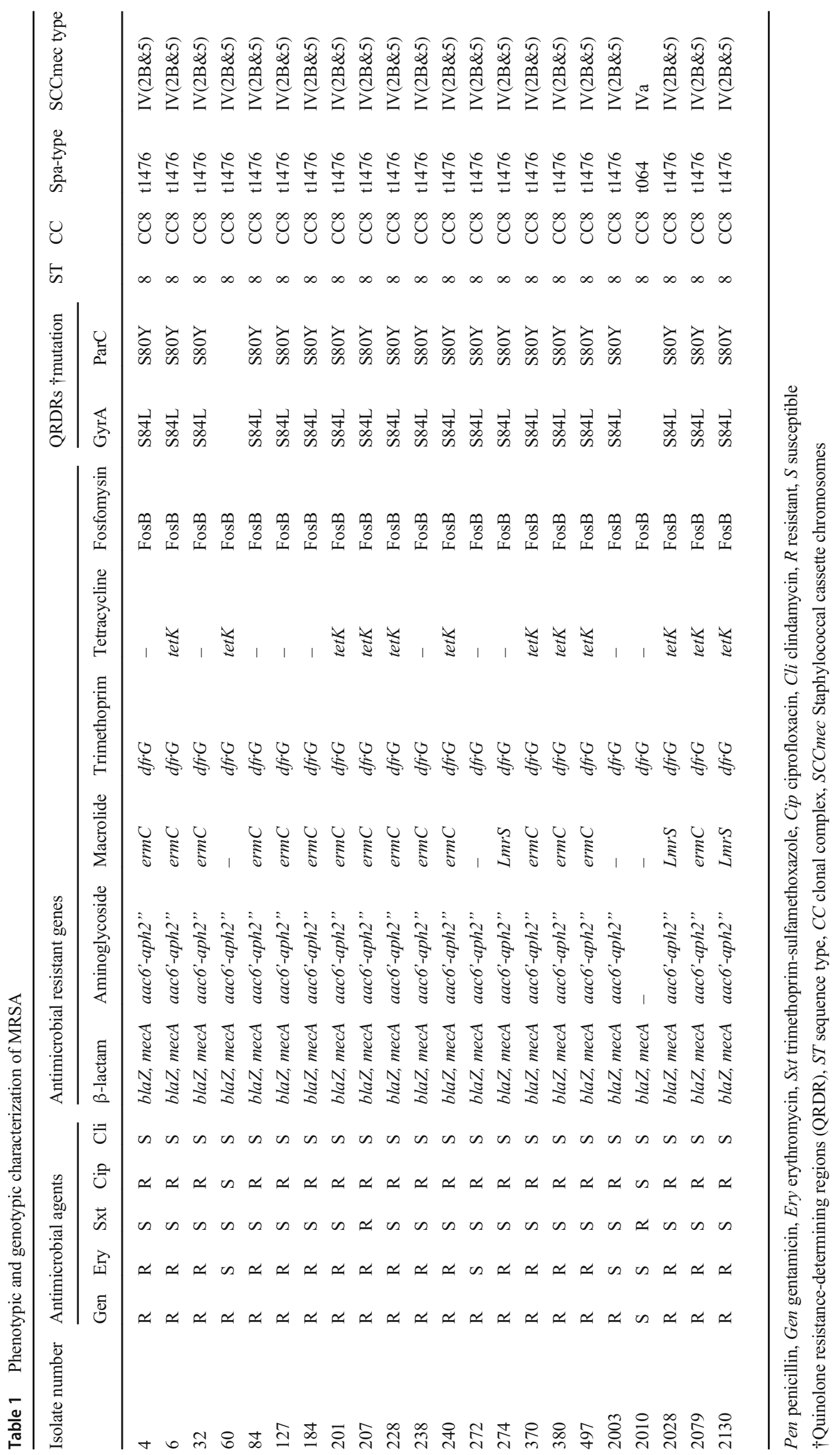


Table 2 Virulence Genes of MRSA

\begin{tabular}{|c|c|c|c|}
\hline PID number & Toxin & Exo-enzyme & IEC \\
\hline 4 & $\operatorname{lukD}, \operatorname{lukE}, h \lg A, h \lg B, h g l C, \operatorname{sej}, \mathrm{ser}$ & aur, splA, splB, slpE & sak, scn \\
\hline 6 & $\operatorname{lukD}, \operatorname{lukE}, h \lg A, h \lg B, h g l C$, sej, ser & aur, splA, splB, slpE & sak, scn \\
\hline 32 & $\operatorname{lukD}, \operatorname{lukE}, h \lg A, h \lg B, h g l C$, sej, ser & aur, splA, splB, slpE & sak, sch \\
\hline 60 & $\operatorname{lukD}, \operatorname{lukE}, h \lg A, h \lg B, h g l C$ & aur, splA, splB, slpE & sak, sch \\
\hline 84 & $\operatorname{lukD}, \operatorname{lukE}, h \lg A, h \lg B, h g l C, \operatorname{sej}, \mathrm{ser}$ & aur, splA, splB, slpE & sak, sch \\
\hline 127 & $\operatorname{lukD}, \operatorname{lukE}, h \lg A, h \lg B, h g l C$, sej, ser & aur, splA, splB & sak, sch \\
\hline 184 & $\operatorname{lukD}, \operatorname{lukE}, h \lg A, h \lg B, h g l C$, sej, ser & aur, splA, splB & sak, sch \\
\hline 201 & $\operatorname{lukD}, \operatorname{lukE}, h \lg A, h \lg B, h g l C$, sej, ser & aur, splA, splB, slpE & sak, sch \\
\hline 207 & $\operatorname{lukD}, \operatorname{lukE}, h \lg A, h \lg B, h g l C$, sej, ser & aur, splA, splB, slpE & sak, sch \\
\hline 228 & $\operatorname{lukD}, \operatorname{lukE}, h \lg A, h \lg B, h g l C$, sej, ser & aur, splA, splB, slpE & sak, sch \\
\hline 238 & $\operatorname{lukD}, \operatorname{lukE}, h \lg A, h \lg B, h g l C$, sej, ser & aur, splA, splB & sak, sch \\
\hline 240 & lukD, lukE, hlgA, hlgB, hglC, sec sej, sel, ser & aur, splA, splB & sak, sch \\
\hline 272 & $\operatorname{lukD}, \operatorname{lukE}, h \lg A, h \lg B, h g l C, \operatorname{sej}, \mathrm{ser}$ & aur, splA, splB & sak, sch \\
\hline 274 & $\operatorname{lukD}, \operatorname{lukE}, h \lg A, h \lg B, h g l C$, sej, ser & aur, splA, splB, slpE & sak, sch \\
\hline 370 & $\operatorname{lukD}, \operatorname{lukE}, h \lg A, h \lg B, h g l C$, sej, ser & aur, splA, splB, slpE & sak, sch \\
\hline 380 & lukD, lukE, hlgA, hlgB, hglC, sej, ser & aur, splA, splB, slpE & sak, sch \\
\hline 497 & $\operatorname{lukD}, \operatorname{lukE}, h \lg A, h \lg B, h g l C$, sej, ser & aur, splA, splB, slpE & sak, scn \\
\hline 2003 & $\operatorname{lukD}, \operatorname{lukE}, \mathrm{hlg} A, h \lg B, h g l C, \mathrm{sej}, \mathrm{ser}$ & aur, splA, splB, slpE & sak, sch \\
\hline 2010 & lukD, lukE, hlgA, hlgB, hglC, sea, seb, sek, seq & aur, splA, splB, slpE & sak, sch \\
\hline 2028 & $\operatorname{lukD}, \operatorname{lukE}, \mathrm{hlg} A, h \lg B, h g l C, \operatorname{sej}, \mathrm{ser}, \mathrm{tst}$ & aur, splA, splB, slpE & sak, sch \\
\hline 2079 & lukD, lukE, hlgA, hlgB, hglC, sej, seq, ser & aur, splA, splB, slpE & sak, sch \\
\hline 2130 & $\operatorname{lukD}, \operatorname{lukE}, h \lg A, h \lg B, h g l C, \operatorname{sej}, \mathrm{ser}$ & aur, splA, splB, slpE & sak, sch \\
\hline
\end{tabular}

IEC Immune evasion cluster, $l u k$ leucocidin, $h \lg A$ gamma-hemolysin chain II precursor, $h \lg B$ gamma-hemolysin component B precursor, $h \lg C$ gamma-hemolysin component $\mathrm{C}$ precursor, sea enterotoxin, sea enterotoxin A seb enterotoxin B, sec enterotoxin C, sej enterotoxin J, sek enterotoxin K, ser enterotoxin R, seq enterotoxin Q, aur aureolysin, $s p l$ serine protease, sak Staphylokinase, scn Staphylococcal complement inhibitor, tst toxic shock syndrome toxin-1

\section{Discussion}

This is the first description of whole genome sequencing data from MRSA isolates from HIV-infected adults in Tanzania and also the first report of nasal/nasopharyngeal MRSA colonization among newly diagnosed HIV-infected adults in Tanzania. Overall, four percent of all participants were nasal/ nasopharyngeal MRSA carriers, and all but one belonged to lineage ST8-SCCmecIV-t1476. These isolates were collected in the span of 2 years, from six HIV care and treatment centers located in different municipalities in Dar es Salaam. This finding suggests predominance and wide spread of the ST8 CAMRSA (non-USA300) clone in the HIV-infected population in Dar es Salaam, Tanzania. Diverse lineages of ST8 CA-MRSA dominate different regions in the world. However, the ST8SCCmecIV (USA300) clone has successfully spread in HIVinfected populations in the developed world [17] and is commonly associated with skin and soft tissue infections [18]. No previous study from East Africa has characterized MRSA isolates from HIV-infected population using whole genome sequencing. The only other study in Tanzania, unveiling characteristics of MRSA clinical isolates by whole genome sequencing, found six out of ten MRSA which were ST8 (non-USA), followed by two ST239 and two had unknown sequence type [12]. However, this study did not describe the SCCmec and spa-type of those isolates.

The ST8-spa-t1476 CA-MRSA has rarely been reported outside the African continent, and the types of infection it causes are not known. It has been reported once in the UK in an outbreak of patients with no evidence of links to the African region [19]. The ST8-SCCmecV-t1476 clone has been frequently reported among CA-MRSA in the Democratic Republic of Congo (DRC) [20, 21]. Our ST8-SCCmecIV MRSA isolates had close resemblance to those circulating in DRC, despite carrying different staphylococcal cassette chromosomes $[20,21]$. There is evidence that MSSA acts as reservoir for MRSA before acquisition of staphylococcal cassette chromosomes [22]. Isolates from Tanzania and DRC may have shared common genetic ancestors before each acquiring different SCCmec.

On phylogenetic analysis, all ST8-SCCmecIV-t1476 from the present study were clustered into one clade and might have shared a common ancestor and were not related to already known ST8 (USA300) clones from the USA and Africa. 
Fig. 1 SNP phylogenetic tree for ST8 MRSA isolates. Blue indicates MRSA isolated from this study, pink indicates MRSA isolated previously from Tanzania, black are MRSA isolated from USA at different point of time, and green is an isolate from Gabon
Tree scale: 0.1

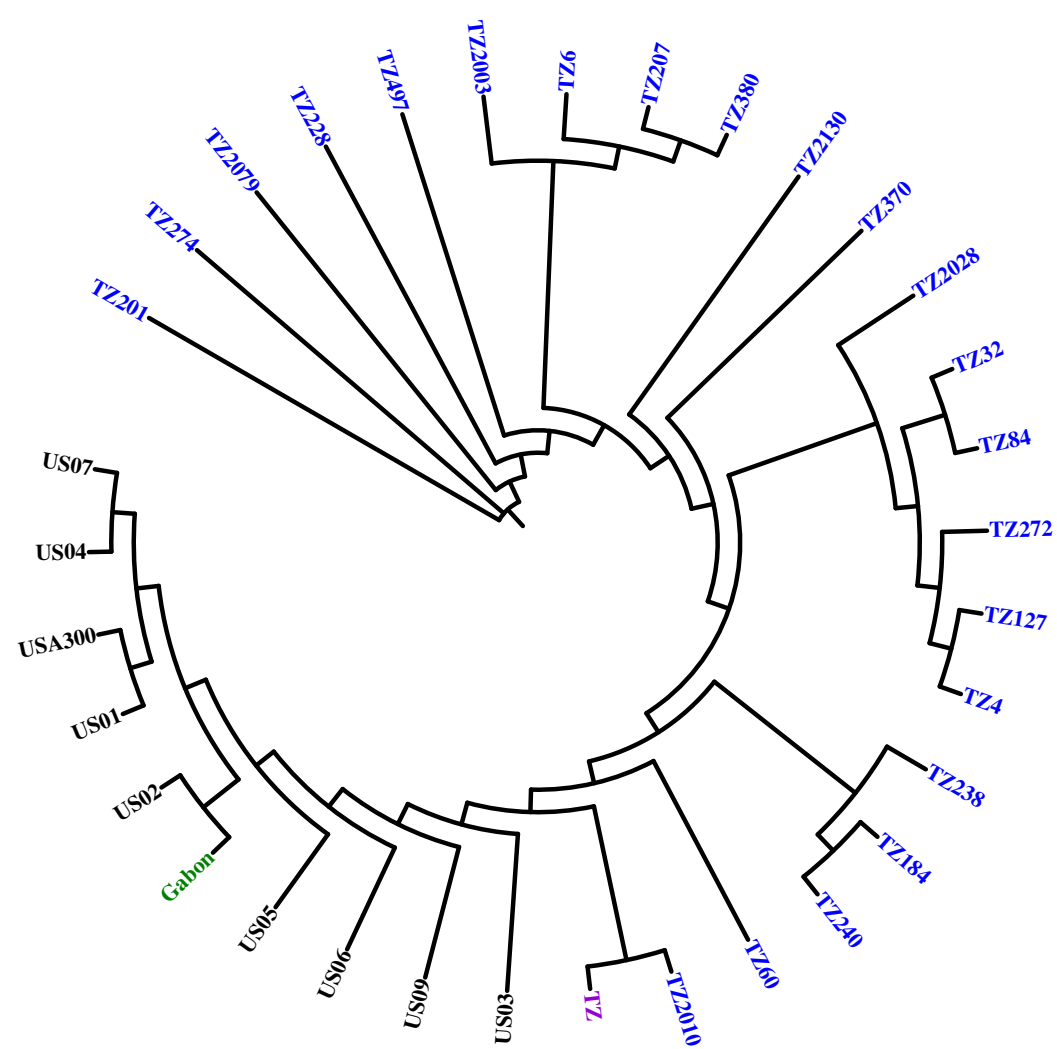

One isolate from the present study was clustered in a clade with already known ST8 clone, but SNPs analysis showed they were not related. Our findings demonstrate that the epidemiology of the ST8 CA-MRSA clone lineages varies remarkably in different regions of the world. Evolutionary studies have shown that all ST8 clones have a common ancestor but may subsequently acquire certain characteristics like PVL, ACME, and SCCmec-type [23]. Repeated introduction of one ST8 CA-MRSA clone in a geographic area may replace existing clones. Our finding and previous data from East and Central Africa confirms predominance of non-USA300 clones $[20,21]$. A previous evolutionary study involving ST8 MRSA clones from different continents found that no African ST8 isolates had direct ancestry to the USA300 clades [23]. Further studies are needed to understand the origin and evolution of this clone in our region.

MRSA isolated from newly diagnosed HIV-infected patients were also resistant to gentamicin, erythromycin, and ciprofloxacin but showed low rate of resistance to trimethoprimsulfamethoxazole phenotypically. However, the presence of the resistance gene $d$ fr $G$ in all isolates phenotypically susceptible to trimethoprim-sulfamethoxazole in our study, suggests that this antibiotic could be suboptimal for treatment of MRSA infections. In case it is used, careful clinical monitoring is needed to avoid treatment failure, since in vitro susceptibility might not be matched by susceptibility in vivo.
The present study demonstrates that vancomycin and linezolid could be used for treatment of MRSA infections; however, these drugs are not widely available in East Africa. Macrolides and lincosamides are commonly used in treatment of staphylococcal skin and soft tissue infections. MRSA resistant to macrolides, lincosamides, and streptogramin type B are mainly mediated by erm $\mathrm{A}$ and erm $\mathrm{C}$, which codes for erythromycin ribosomal methylase [24]. We observed predominance of ermC from our MRSA isolates (83\%), and no isolates carried ermA. Previous studies in Tanzania and the DRC reported similar predominance of ermC among ST8 CAMRSA [12, 25]. The observation of $73 \%$ inducible clindamycin resistance among MRSA implies that clindamycin may not be a reliable treatment option for infections caused by MRSA. In such cases, treatment with clindamycin may induce clinical resistance in apparently susceptible isolates harboring erm $\mathrm{C}$ genes [26], leading to clinical treatment failure.

Gentamicin resistance in MRSA was mediated by aac6'aph2" and ciprofloxacin resistance by mutations in the QRDR sequence of the gyrA and parC genes. This finding is similar to other studies among ST8 CA-MRSA from East Africa and Europe [12, 27, 28]. Although fosfomycin, an older broad spectrum antibiotic [29], has not been commonly used in Tanzania, we still found $100 \%$ of MRSA carrying genes conferring resistance to fosfomycin. The high prevalence of FosB 
in the CA-MRSA isolates is surprising considering the absence of selective pressure by actual use of fosfomycin. This finding could be accounted for by high consumption of other antibiotics including tetracycline which may select for fosfomycin resistance or by the emergence of spontaneous mutation [30, 31].

The ability of MRSA to cause invasive disease depends on virulence factors. Remarkably, the PVL, which is usually present in ST8 CA-MRSA, was lacking from the MRSA isolates in our study. However, several virulence factors were identified including immune evasion cluster, gamma-hemolysins ( $h l g A$, $h \lg B, h \lg C$ ), enterotoxin, and leucocidin ED. Recently, an animal model has shown that leucocidin ED contributes to systemic infection by targeting neutrophils and promoting bacterial growth in vivo [32]. The immune evasion gene cluster enhances capacity of $S$. aureus to colonize, disseminate and persist in a human host [33]. The present study demonstrates that MRSA colonizing the nose/nasopharynx of HIV-infected individuals may have a significant potential to cause invasive disease due to the variety of virulence factors observed.

One caveat of our study is that, although only one of our participants had a history of recent hospitalization and five had visited outpatient clinics, we cannot rule out that MRSA in these patients could have been hospital acquired. However, the fact that all MRSA had the same SCCmecIV type which is commonly found in community-acquired strains supports the notion that the MRSA isolates were community acquired. The anterior nares are a common place of staphylococcal colonization, and swabbing this site is appropriate to detect the bacteria. We swabbed the nasopharynx first, and the anterior nares were swabbed afterwards while retracting the swab. This procedure may have led to falsely low prevalence of $S$. aureus, which could be a limitation of the study.

\section{Conclusion}

ST8 CA-MRSA (non-USA300) was found to be the commonest circulating population structure in newly diagnosed HIV-infected adults in Tanzania. The circulating ST8 CA-MRSA isolates were not related to other common, successful circulating ST8 lineages. The spa-type t1476 is predominant in this CA-MRSA. The majority of CA-MRSA was highly resistant to non-beta lactam antibiotics. Screening for colonization of MRSA and intervention in HIV-infected outpatients and inpatients may control the spread of the strain.

Acknowledgments The authors acknowledge members of the Department of Microbiology and Immunology, Muhimbili University of Health and Allied Science, Dar es Salaam, Tanzania, for their support during data collection and initial laboratory procedures.

Authors' contributions BB, NL, and SM conceived the study. All authors contributed to designing the study. JM collected study data. JM and SM performed the microbiological investigations. BB and JM performed statistical analysis. JM drafted the manuscript. SA, BB, SM, and NL revised the manuscript. All authors approved the final version.

Funding This study was funded by the Helse Bergen HF, Haukeland University Hospital, Norway, through project number 912132 and by the National Advisory Unit on Tropical Infectious Diseases, Haukeland University Hospital. The funders had no role in study design, data collection and analysis, decision to publish, or preparation of the manuscript.

Data availability Data are available on request.

\section{Compliance with ethical standards}

Competing interests The authors declare that they have no competing interests.

Ethical approval Approvals to conduct this study in Tanzania were obtained from Muhimbili University of Heath and Allied Sciences Senate Research and Publications Committee (Ref. No. 2015-10-27/ AEC/Vol.X/54), National Health Research Ethics Committee (Ref. No. NIMRIHQ/R. SaJVol. 1X12144), Tanzania Medicines and Medical Devices Authority (Ref. No. TZ16CT007), and Regional Committee for Medical and Health Research Ethics of Western Norway (Ref. No. REK2015/540).

Informed consent Written informed consent was obtained from each study participant prior to the enrolment in the study.

Consent to publish Consent to publish was obtained from National Health Research Ethics Committee (Ref. No. NIMRIHQ/R. SaJVol. 1X12144).

Open Access This article is licensed under a Creative Commons Attribution 4.0 International License, which permits use, sharing, adaptation, distribution and reproduction in any medium or format, as long as you give appropriate credit to the original author(s) and the source, provide a link to the Creative Commons licence, and indicate if changes were made. The images or other third party material in this article are included in the article's Creative Commons licence, unless indicated otherwise in a credit line to the material. If material is not included in the article's Creative Commons licence and your intended use is not permitted by statutory regulation or exceeds the permitted use, you will need to obtain permission directly from the copyright holder. To view a copy of this licence, visit http://creativecommons.org/licenses/by/4.0/.

\section{References}

1. Cosgrove SE, Sakoulas G, Perencevich EN, Schwaber MJ, Karchmer AW, Carmeli Y (2003) Comparison of mortality associated with methicillin-resistant and methicillin-susceptible Staphylococcus aureus bacteremia: a meta-analysis. Clin Infect Dis 36(1):53-59

2. Stenehjem E, Rimland D (2013) MRSA nasal colonization burden and risk of MRSA infection. Am J Infect Control 41(5):405-410

3. Szumowski JD, Wener KM, Gold HS, Wong M, Venkataraman L, Runde CA, Cohen DE, Mayer KH, Wright SB (2009) Methicillinresistant Staphylococcus aureus colonization, behavioral risk factors, and skin and soft-tissue infection at an ambulatory clinic serving a large population of HIV-infected men who have sex with men. Clin Infect Dis 49(1):118-121 
4. Madhi SA, Petersen K, Madhi A, Khoosal M, Klugman KP (2000) Increased disease burden and antibiotic resistance of bacteria causing severe community-acquired lower respiratory tract infections in human immunodeficiency virus type 1-infected children. Clin Infect Dis 31(1):170-176

5. Naimi TS, LeDell KH, Boxrud DJ, Groom AV, Steward CD, Johnson SK, Besser JM, O'Boyle C, Danila RN, Cheek JE, Osterholm MT, Moore KA, Smith KE (2001) Epidemiology and clonality of community-acquired methicillin-resistant Staphylococcus aureus in Minnesota, 1996-1998. Clin Infect Dis 33(7):990-996

6. Harris SR, Feil EJ, Holden MT, Quail MA, Nickerson EK, Chantratita N, Gardete S, Tavares A, Day N, Lindsay JA, Edgeworth JD, de Lencastre H, Parkhill J, Peacock SJ, Bentley SD (2010) Evolution of MRSA during hospital transmission and intercontinental spread. Science 327(5964):469-474

7. McAdam PR, Templeton KE, Edwards GF, Holden MT, Feil EJ, Aanensen DM, Bargawi HJ, Spratt BG, Bentley SD, Parkhill J, Enright MC, Holmes A, Girvan EK, Godfrey PA, Feldgarden M, Kearns AM, Rambaut A, Robinson DA, Fitzgerald JR (2012) Molecular tracing of the emergence, adaptation, and transmission of hospital-associated methicillin-resistant Staphylococcus aureus. Proc Natl Acad Sci U S A 109(23):9107-9112

8. Ouko TT, Ngeranwa JN, Orinda GO, Bii CC, Amukoye E, Lucy M, Wamae CN (2010) Oxacillin resistant Staphylococcus aureus among HIV infected and non-infected Kenyan patients. East Afr Med J 87(5):179-186

9. Olalekan AO, Schaumburg F, Nurjadi D, Dike AE, Ojurongbe O, Kolawole DO, Kun JF, Zanger P (2012) Clonal expansion accounts for an excess of antimicrobial resistance in Staphylococcus aureus colonising HIV-positive individuals in Lagos, Nigeria. Int $\mathrm{J}$ Antimicrob Agents 40(3):268-272

10. Lemma MT, Zenebe Y, Tulu B, Mekonnen D, Mekonnen Z (2015) Methicillin resistant Staphylococcus aureus among HIV infected pediatric patients in Northwest Ethiopia: carriage rates and antibiotic co-resistance profiles. PLoS One 10(9):e0137254

11. Reid MJA, Steenhoff AP, Mannathoko N, Muthoga C, McHugh E, Brown EL, Fischer RSB (2017) Staphylococcus aureus nasal colonization among HIV-infected adults in Botswana: prevalence and risk factors. AIDS Care 29(8):961-965

12. Kumburu HH, Sonda T, Leekitcharoenphon $\mathrm{P}$, van Zwetselaar M, Lukjancenko O, Alifrangis M, Lund O, Mmbaga BT, Kibiki G, Aarestrup FM (2018) Hospital epidemiology of methicillinresistant Staphylococcus aureus in a tertiary care hospital in Moshi, Tanzania, as determined by whole genome sequencing. Biomed Res Int 2018:2087693

13. Clinical and Laboratory Standards Institute (2018) Performance standards for antimicrobial susceptibility testing; twenty-eighth informational supplement CLSI document M100-S20. Clinical and Laboratory Standards Institute, Wayne, PA

14. Moyo SJ, Aboud S, Blomberg B, Mkopi N, Kasubi M, Manji K, Lyamuya EF, Maselle SY, Langeland N (2014) High nasal carriage of methicillin-resistant Staphylococcus aureus among healthy Tanzanian under-5 children. Microb Drug Resist 20(1):82-88

15. Uhlemann AC, Dordel J, Knox JR, Raven KE, Parkhill J, Holden MT, Peacock SJ, Lowy FD (2014) Molecular tracing of the emergence, diversification, and transmission of $S$. aureus sequence type 8 in a New York community. Proc Natl Acad Sci U S A 111(18): 6738-6743

16. Schaumburg F, Ngoa UA, Kösters K, Köck R, Adegnika AA, Kremsner PG, Lell B, Peters G, Mellmann A, Becker K (2011) Virulence factors and genotypes of Staphylococcus aureus from infection and carriage in Gabon. Clin Microbiol Infect 17(10): $1507-1513$

17. Popovich KJ, Hota B, Aroutcheva A, Kurien L, Patel J, LylesBanks R, Grasso AE, Spec A, Beavis KG, Hayden MK,
Weinstein RA (2013) Community-associated methicillin-resistant Staphylococcus aureus colonization burden in HIV-infected patients. Clin Infect Dis 56(8):1067-1074

18. Srinivasan A, Seifried S, Zhu L, Bitar W, Srivastava DK, Shenep JL, Bankowski MJ, Flynn PM, Hayden RT (2009) Short communication: methicillin-resistant Staphylococcus aureus infections in children and young adults infected with HIV. AIDS Res Hum Retrovir 25(12):1219-1224

19. Utsi L, Pichon B, Arunachalam N, Kerrane A, Batten E, Denton M, Townsend R, Agwuh KN, Hughes GJ, Kearns A (2019) Circulation of a community healthcare-associated multiply-resistant meticillinresistant Staphylococcus aureus lineage in South Yorkshire identified by whole genome sequencing. J Hosp Infect 103(4):454-460

20. De Boeck H, Vandendriessche S, Hallin M, Batoko B, Alworonga JP, Mapendo B, Van Geet C, Dauly N, Denis O, Jacobs J (2015) Staphylococcus aureus nasal carriage among healthcare workers in Kisangani, the Democratic Republic of the Congo. Eur J Clin Microbiol Infect Dis 34(8):1567-1572

21. Vandendriessche S, De Boeck H, Deplano A, Phoba MF, Lunguya O, Falay D, Dauly N, Verhaegen J, Denis O, Jacobs J (2017) Characterisation of Staphylococcus aureus isolates from bloodstream infections, Democratic Republic of the Congo. Eur J Clin Microbiol Infect Dis 36(7):1163-1171

22. Driebe EM, Sahl JW, Roe C, Bowers JR, Schupp JM, Gillece JD, Kelley E, Price LB, Pearson TR, Hepp CM, Brzoska PM, Cummings CA, Furtado MR, Andersen PS, Stegger M, Engelthaler DM, Keim PS (2015) Using whole genome analysis to examine recombination across diverse sequence types of Staphylococcus aureus. PLoS One 10(7):e0130955

23. Strauss L, Stegger M, Akpaka PE, Alabi A, Breurec S, Coombs G, Egyir B, Larsen AR, Laurent F, Monecke S, Peters G, Skov R, Strommenger B, Vandenesch F, Schaumburg F, Mellmann A (2017) Origin, evolution, and global transmission of communityacquired Staphylococcus aureus ST8. Proc Natl Acad Sci U S A 114(49):E10596-e10604

24. Khodabandeh M, Mohammadi M, Abdolsalehi MR, Alvandimanesh A, Gholami M, Bibalan MH, Pournajaf A, Kafshgari R, Rajabnia R (2019) Analysis of resistance to macrolide-Lincosamide-Streptogramin B among mecA-positive Staphylococcus aureus isolates. Osong Public Health Res Perspect 10(1):25-31

25. Phaku P, Lebughe M, Strauss L, Peters G, Herrmann M, Mumba D, Mellmann A, Muyembe-Tamfum JJ, Schaumburg F (2016) Unveiling the molecular basis of antimicrobial resistance in Staphylococcus aureus from the Democratic Republic of the Congo using whole genome sequencing. Clin Microbiol Infect 22 (7):644.e641-645

26. Steward CD, Raney PM, Morrell AK, Williams PP, McDougal LK, Jevitt L, McGowan JE Jr, Tenover FC (2005) Testing for induction of clindamycin resistance in erythromycin-resistant isolates of Staphylococcus aureus. J Clin Microbiol 43(4):1716-1721

27. Kyany'a C, Nyasinga J, Matano D, Oundo V, Wacira S, Sang W, Musila L (2019) Phenotypic and genotypic characterization of clinical Staphylococcus aureus isolates from Kenya. BMC Microbiol 19(1):245

28. Enström J, Fröding I, Giske CG, Ininbergs K, Bai X, Sandh G, Tollström UB, Ullberg M, Fang H (2018) USA300 methicillinresistant Staphylococcus aureus in Stockholm, Sweden, from 2008 to 2016. PLoS One 13(11):e0205761

29. Raz R (2012) Fosfomycin: an old-new antibiotic. Clin Microbiol Infect 18(1):4-7

30. McCaughey G, Diamond P, Elborn JS, McKevitt M, Tunney MM (2013) Resistance development of cystic fibrosis respiratory pathogens when exposed to fosfomycin and tobramycin alone and in combination under aerobic and anaerobic conditions. PLoS One 8(7):e69763 
31. Sahuquillo Arce JM, Colombo Gainza E, Gil Brusola A, Ortiz Estévez R, Cantón E, Gobernado M (2006) In vitro activity of linezolid in combination with doxycycline, fosfomycin, levofloxacin, rifampicin and vancomycin against methicillinsusceptible Staphylococcus aureus. Rev Esp Quimioter 19(3): 252-257

32. Alonzo F 3rd, Benson MA, Chen J, Novick RP, Shopsin B, Torres VJ (2012) Staphylococcus aureus leucocidin ED contributes to systemic infection by targeting neutrophils and promoting bacterial growth in vivo. Mol Microbiol 83(2):423-435
33. van Wamel WJ, Rooijakkers SH, Ruyken M, van Kessel KP, van Strijp JA (2006) The innate immune modulators staphylococcal complement inhibitor and chemotaxis inhibitory protein of Staphylococcus aureus are located on beta-hemolysin-converting bacteriophages. J Bacteriol 188(4):1310-1315

Publisher's note Springer Nature remains neutral with regard to jurisdictional claims in published maps and institutional affiliations. 